\title{
Psoriasis verursacht hohe Kosten, mindert die Produktivität am Arbeitsplatz und verringert die Lebensqualität
}

\author{
Psoriasis Causes High Costs, Reduces Productivity at Work and Quality of Life
}

Autoren

Institute
M. Augustin ${ }^{1}$, J. Chapnik ${ }^{2}$, S. Gupta ${ }^{2}$, K. Buesch ${ }^{3}$, M. Radtke ${ }^{1}$

CVderm, Institut für Versorgungsforschung in der Dermatologie und bei Pflegeberufen, Universitätsklinikum Hamburg-Eppendorf

2 Consumer Health Sciences, Princeton/USA

Abbott GmbH \& Co. KG, Global Health Economics \& Outcomes Research, Ludwigshafen am Rhein
Bibliografie

DOI http://dx.doi.org/ 10.1055/s-0030-1256636 Akt Dermatol 2011; 37: 353-359 ๔ Georg Thieme Verlag KG Stuttgart · New York ISSN 0340-2541

Korrespondenzadresse
Dr. med. Univ.-Prof.
Matthias Augustin
Competenzzentrum
Versorgungsforschung in der
Dermatologie (CVderm)
Institut für Versorgungs-
forschung in der Dermatologie
und bei Pflegeberufen (IVDP)
Universitätsklinikum
Hamburg-Eppendorf
Martinistr. 52
20246 Hamburg
m.augustin@uke.de

m.augustin@uke.de

\section{Zusammenfassung \\ $\nabla$}

Hintergrund: Trotz ihrer großen medizinischen und ökonomischen Bedeutung wurden zur Psoriasis vulgaris (Pso) in Deutschland bisher nur wenige bevölkerungsbezogene Daten erhoben. Material und Methoden: In einer webbasierten Gesundheitsstudie (NHWS = National Health and Wellness Survey) wurden 2007 bundesweit erwachsene Pso-Patienten und Nicht-Betroffene zu medizinischer Ressourcennutzung, Arbeitsproduktivität und gesundheitsbezogener Lebensqualität (LQ) befragt.

Ergebnisse: Von den 15000 Befragten gaben 382 Personen $(2,5 \%)$ die ärztlich bestätigte Diagnose

\section{Einleitung \\ $\nabla$}

Psoriasis vulgaris (Pso) ist eine chronisch-entzündliche Systemerkrankung, von der weltweit ca. 1,5-3\% der Bevölkerung betroffen sind [11]. Für Deutschland wurde eine Punktprävalenz von $2,0 \%$ und eine Jahresprävalenz von $2,5 \%$, bei Kindern von $0,71 \%$ publiziert $[28,1,2]$. Dies entspricht bundesweit ca. zwei Millionen betroffenen Menschen [28]. Die Pso gehört zum Formenkreis der immunologisch vermittelten Erkrankungen und ist mit Komorbiditäten wie der Psoriasis-Arthritis (PsA), dem metabolischen Syndrom und kardiovaskulären Krankheiten assoziiert [3,24]. Eine erhöhte Komorbidität wurde auch bei Kindern gezeigt [1]. Diese Erkrankungen erhöhen maßgeblich das Mortalitätsrisiko, bei schwerer Pso in bestimmten Altersgruppen sogar auf bis zu 50\% [10]. Eine erhöhte Komorbidität wurde auch bei Kindern gezeigt [1]. Aufgrund ihrer hohen gesundheitsökonomischen wie medizinischen Bedeutung war die Psoriasis in den letzten Jahren Gegenstand intensiver Versorgungsforschung [4]. Auch wurden in Deutschland mehrere Krankheitskostenstudien durchgeführt $[5,30]$. Hier
Pso an. Davon waren $72 \%(n=264)$ von einer leichten und $28 \%(n=118)$ von einer mittelschweren oder schweren Form betroffen. Befragte mit mittelschwerer/schwerer Pso nahmen in stärkerem Umfang die Ressourcen des Gesundheitssystems in Anspruch. Außerdem hatten diese fast doppelt so hohe eigene finanzielle Aufwendungen. Ihre Arbeitsproduktivität sowie ihre LQ waren signifikant vermindert.

Schlussfolgerung: Pso führt zu erhöhten Ressourcenverbräuchen im Gesundheitssystem, verminderter Lebensqualität und reduzierter $\mathrm{Ar}$ beitsproduktivität. Diese Krankheitsfolgen sind für die Therapieplanung und Versorgung von großer Bedeutung.

zeigte sich, dass neben den direkten Kosten aufgrund von Arztbesuchen, Arzneimittelverordnungen und Krankenhausaufenthalten auch relevante indirekte Kosten (z.B. durch krankheitsbedingte Arbeitsunfähigkeit, Leistungsminderung oder Frühberentung) entstehen [5,32]. Nicht nur der Schweregrad der Erkrankung, sondern auch die subjektiv empfundene Krankheitslast korrespondiert mit den Kosten [13]. Pso beeinflusst den Alltag, das Arbeits- und Sozialleben sowie das Selbstwertgefühl der Patienten deutlich negativ $[13,29,36]$. Berichtet wurde über Probleme am Arbeitsplatz und in den sozialen Beziehungen, soziale Stigmatisierung [29] und Suizidalität. Dabei korreliert der Grad der psychosozialen Last z.T. nur mäßig mit dem klinischen Schweregrad der Erkrankung [7,12,27].

Die Versorgungsqualität und Ökonomie der Psoriasis wurde bisher überwiegend durch Studien in dermatologischen Praxen und Kliniken mit den entsprechenden Selektionsnachteilen beschrieben [5,21,22,30]. Bevölkerungsbezogene Daten zur Psoriasis konnten in Deutschland bislang nur aus Sekundärdatenstudien der GKV [3] oder aus einer Kohorte Gesunder im Zuge von 
Hautkrebs-Screenings [28] bezogen werden. Bei beiden wurden aber keine ökonomischen und klinischen Daten sowie Angaben zur Lebensqualität erhoben.

Mit der vorliegenden Studie sollte erstmals in Deutschland eine bevölkerungsbezogene Stichprobe von Patienten mit Psoriasis vulgaris zu den ökonomischen und klinischen Merkmalen der Erkrankung befragt werden.

\section{Fragestellung}

- Wie häufig kommt Psoriasis vulgaris in einer zufälligen Bevölkerungsstichprobe in Deutschland vor?

- Wie unterscheiden sich Personen mit Pso von Nicht-Betroffenen hinsichtlich

- medizinischer Ressourcennutzung,

- Arbeitsproduktivität und

- gesundheitsbezogener LQ?

Wie unterscheiden sich Pso-Patienten mit unterschiedlichen Schweregraden der Erkrankung in Bezug auf diese drei Dimensionen untereinander?

\section{Studiendesign \\ $\nabla$}

Internetbasierte Kohortenstudie auf der Basis der in Deutschland erhobenen Daten einer zwischen Juni und August 2007 durchgeführten länderübergreifenden Umfrage (NHWS, National Health and Wellness Survey). Die NHWS ist eine jährlich durchgeführte Studie, in der die Teilnehmer ( $\geq 18$ Jahre) per Internet zu Erkrankungen, medizinischer Ressourcennutzung, Lebensqualität und sozialmedizinischen Faktoren in mehr als hundert Therapiefeldern befragt werden. Aus der für Deutschland repräsentativen Stichprobe (stratifiziert nach Alter und Geschlecht) wurden diejenigen Befragten in einer Gruppe zusammengefasst, die angaben, an einer ärztlich diagnostizierten Psoriasis zu leiden. Da die NHWS nicht indikationsbezogen ist, wurde aus den nicht von Pso betroffenen Personen die Vergleichsgruppe gebildet.

\section{Schweregrad der Pso \\ $\nabla$}

Der Schweregrad der Pso wurde entsprechend der selbstberichteten betroffenen Körperoberfläche BSA (Body Surface Area) unterteilt in [9]:

- Leichte Pso: $<2 \%$ BSA

- Mittelschwere Pso: 2-10\% BSA

- Schwere Pso: > 10\% BSA.

Aufgrund der geringen Fallzahl in der Gruppe der schwer betroffenen Patienten wurden diese für die statistische Auswertung mit den mittelschwer Betroffenen zu einer Gruppe zusammengefasst. Für alle statistischen Vergleiche wurde das Signifikanzniveau auf 5\% (zweiseitig) festgelegt. Um auch die Belastung in der Gruppe der schwer Betroffenen aufzuzeigen, wurden die Daten dieser Gruppe - obwohl eine Signifikanztestung nicht möglich war - zusätzlich getrennt dargestellt.

\section{Komorbidität}

$\nabla$

Die Häufigkeit von Komorbiditäten wurde auf der Basis der Patientenangaben erhoben und den Angaben der Kontrollgruppe (d.h. der nicht von Psoriasis betroffenen Bevölkerung) gegenübergestellt.

\section{Ressourcenverbräuche und Kosten}

$\nabla$

Als direkte Kosten(-faktoren) wurden die Arztbesuche und Klinikaufenthalte sowie die patientenseitigen Krankheitskosten dokumentiert.

\section{Arbeitsproduktivität}

$\nabla$

Zur Bezifferung von Arbeitsausfall und Produktivitätsverlust wurde der Work Productivity and Activity Impairment (WPAI)Fragebogen verwendet [25].

Der WPAI erlaubt es, krankheitsbedingte Fehlzeiten und Verminderung von Arbeitsproduktivität sowie kombinierte Effekte dieser beiden Dimensionen von Produktivitätseinbußen (Total Work Productivity Index; TWPI) prozentual zur möglichen Produktivität zu beziffern. Der Total Activity Index (TAI) gibt an, inwieweit ein Befragter in seinen alltäglichen, außerberuflichen Tätigkeiten eingeschränkt war. Höhere Werte signalisieren eine stärkere Beeinträchtigung.

\section{Lebensqualität}

Die Fragen an die Teilnehmer bezogen sich auf deren allgemeine Gesundheit und wurden somit nicht Pso-spezifisch gestellt. Als validierter Fragebogen wurde der Short Form 12 Version 2 (SF12v2) zur Bewertung der gesundheitsbezogenen LQ eingesetzt [6]. Die Studienteilnehmer gaben darin selbst Auskunft bzgl. ihrer körperlichen und psychischen Gesundheit sowie ihrer allgemeinen LQ. Aus den Antworten wurden zwei Summenskalen berechnet, die die jeweilige Krankheitslast in der körperlichen (Physical Component Score; PCS) und in der psychischen Dimension (Mental Component Score; MCS) quantifizierten. Scores über 50 weisen auf eine bzgl. der Norm erhöhte LQ hin; Scores unter 50 zeigen eine verminderte LQ an [35].

\section{Statistische Analyse \\ $\nabla$}

Die erhobenen Daten wurden anhand der für Deutschland bekannten Populationsverteilung nach Geschlecht und Alter gewichtet und die Häufigkeit der Pso und ihrer unterschiedlichen Schweregrade auf die Gesamtbevölkerung hochgerechnet [34]. Die statistische Signifikanz wurde bei Prozentdifferenzen mit Hilfe des Z-Tests und bei Mittelwertunterschieden mit dem Student-T-Test ermittelt.

\section{Ergebnisse \\ $\nabla$}

In der für Deutschland repräsentativen Stichprobe von 15000 Befragten gaben $\mathrm{n}=417$ Personen $(2,8 \%)$ an, an Pso zu leiden (๑ Abb. 1).

Bei $n=382$ Befragten (2,5\%) war diese Diagnose durch einen Arzt bestätigt worden, sodass nur diese Personen als Pso-Erkrankte gewertet wurden. Diese Gruppe setzte sich zusammen aus 264 Betroffenen mit einer leichten, 90 Personen mit einer mittelschweren und 28 Personen mit einer schweren Pso.

Hochgerechnet auf Deutschland entspräche die Prävalenz von 2,5\% bei einer erwachsenen Bevölkerung von 68,1 Millionen 1,9 Millionen von Pso betroffenen Erwachsenen. Von diesen litten 


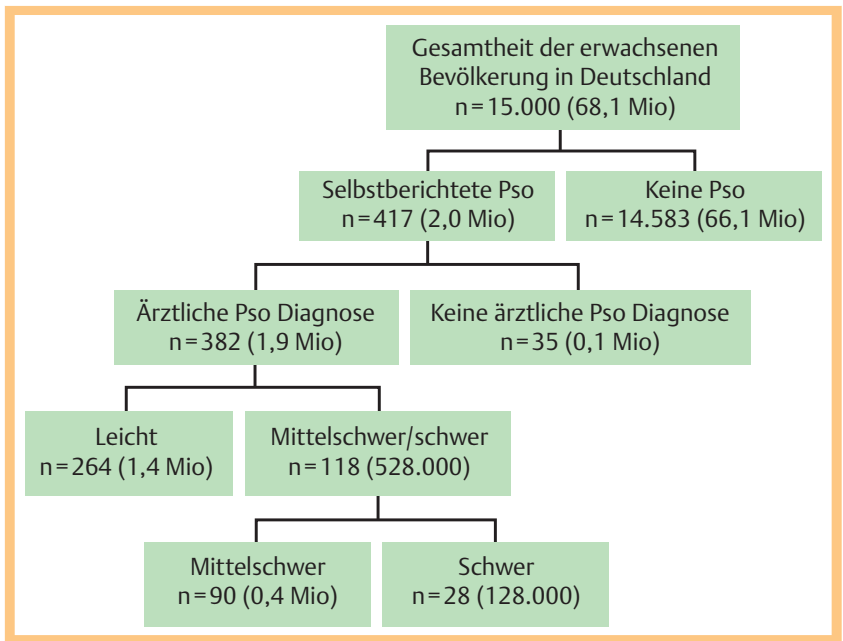

Abb. 1 Auswahl der Stichproben (NHWS) und projizierte Prävalenz der Pso in Deutschland; Pso = Psoriasis vulgaris.

1,4 Millionen Personen (72\%) an einer leichten und 528000 $(28 \%)$ an einer mittelschweren/schweren Form, wenn die befallene Körperoberfläche als Schweregradmaß verwendet würde.

\section{Demografische und krankheitsbezogene Daten}

Die Patienten mit Pso waren durchschnittlich fünf Jahre älter als Befragte ohne Pso (54,2 vs. 49,2 Jahre; $\mathrm{p}<0,05$, $\odot$ Tab. 1).

Mittelschwer bis schwer Betroffene wiesen dagegen ein signifikant niedrigeres Alter als Befragte mit leichter Pso auf (50,5 vs. 55,7 Jahre; $\mathrm{p}<0,05$ ). Männer und Frauen waren etwa gleich häufig betroffen.

In der Gruppe der mittelschwer/schwer Betroffenen waren knapp 50\% mehr Befragte adipös als in der Gruppe der Nicht-Betroffenen $(p<0,05)$. Bei den schwer betroffenen Pso-Patienten gaben sogar $40 \%$ der Befragten einen Body Mass Index (BMI) von mindestens 30 an.

Personen mit Pso wiesen einen signifikant (jeweils $p<0,05$ ) höheren Anteil an ärztlich diagnostizierten Komorbiditäten auf für Arthritis (12,7\% vs. 5,2\%), Diabetes mellitus (20,0\% vs. $7,4 \%$ ) und Depression (14,2\% vs. 8,0\%). Auch der Anteil an Personen mit Schlafstörungen war bei Pso mit 22,8\% vs. 11,6\% signifikant höher $(\mathrm{p}<0,05)$.

\section{Nutzung der Ressourcen des Gesundheitswesens}

Personen mit Pso suchten signifikant häufiger einen Arzt auf als Befragte ohne Pso (96\% vs. 87\%; p < 0,05; ๑ Tab. 2).

Tab. 1 Demografische und krankheitsbezogene Daten.

\begin{tabular}{|c|c|c|c|c|c|}
\hline & Keine Pso & Pso gesamt & Leichte Pso & $\begin{array}{l}\text { Mittelschwere/ } \\
\text { Schwere Pso }\end{array}$ & Schwere Pso \\
\hline $\mathrm{N}$ & 14.583 & 382 & 264 & 118 & 28 \\
\hline Alter in Jahren, Mittelwert & 49,2 & $54,2^{*}$ & $55,7^{*}$ & $50,5^{\dagger}$ & 51,5 \\
\hline BMI, Mittelwert & 26,7 & 27,6 & 27,4 & $27,9^{*}$ & 27,7 \\
\hline Adipositas (BMI $\geq 30$ ), in \% & 22 & 24 & 22 & $32^{\circ}$ & 40 \\
\hline Weiblich, in \% & 51 & 58 & 60 & 54 & 50 \\
\hline Erkrankungsdauer in Jahren, Mittelwert & - & 18,8 & 17,9 & 21,0 & 26,3 \\
\hline \multicolumn{6}{|l|}{ Ärztlich diagnostiziert, in \% } \\
\hline Arthritis & 5,2 & $12,7^{*}$ & $10,8^{*}$ & $17,7^{*}$ & 28,5 \\
\hline Diabetes & 7,4 & $20,0^{*}$ & $21,3^{*}$ & $16,7^{*}$ & 25,3 \\
\hline Depression & 8,0 & $14,2^{*}$ & 11,9 & $20,2^{*}$ & 19,7 \\
\hline Schlafstörungen & 11,6 & $22,8^{*}$ & $23,3^{\circ}$ & $21,5^{*}$ & 31,1 \\
\hline Schmerz (letzter Monat), in \% & 27,9 & $42,0^{*}$ & $40,8^{*}$ & $45,1^{*}$ & 52,2 \\
\hline
\end{tabular}

Pso $=$ Psoriasis vulgaris; $\mathrm{BMI}=$ Body Mass Index.

${ }^{*} \mathrm{p}<0,05$ vs. Befragte ohne Pso; ${ }^{\dagger} \mathrm{p}<0,05$ vs. Patienten mit leichter Pso.

¥Verlässliche statistische Schlussfolgerungen konnten aufgrund der geringen Patientenzahl $(<30)$ nicht gezogen werden.

Tab. 2 Ressourcennutzung im Gesundheitssystem in den letzten 6 Monaten.

\begin{tabular}{|c|c|c|c|c|c|}
\hline & Keine Pso & Pso gesamt & Leichte Pso & $\begin{array}{l}\text { Mittelschwere/ } \\
\text { Schwere Pso }\end{array}$ & Schwere Pso $\ddagger$ \\
\hline $\mathrm{N}$ & 14.583 & 382 & 264 & 118 & 28 \\
\hline Arztbesuche, in \% (Anzahl Arztbesuche ${ }^{1}$ ) & $87(7)$ & $96^{*}\left(11^{*}\right)$ & $96^{*}\left(10^{*}\right)$ & $95^{*}\left(13^{*}\right)$ & $97(21)$ \\
\hline Hausarzt & $67(3)$ & $78^{*}\left(4^{*}\right)$ & $78^{*}\left(4^{*}\right)$ & $78^{*}\left(5^{*}\right)$ & $82(9)$ \\
\hline Dermatologe & $8(2)$ & $28^{*}\left(3^{*}\right)$ & $24^{*}(2)$ & $38^{* \dagger}\left(5^{*}\right)$ & $49(8)$ \\
\hline Rheumatologe & $1(2)$ & $7^{*}(3)$ & $5^{*}(3)$ & $13^{* \dagger}(4)$ & $19(2)$ \\
\hline Krankenhausaufenthalt, \% & 9,5 & 14,2 & 15,3 & 11,3 & 8,6 \\
\hline Dauer des KH-Aufenthaltes, in Tagen ${ }^{1}$ & 10,1 & 8,1 & 7,6 & 10,0 & 12,9 \\
\hline
\end{tabular}

Pso $=$ Psoriasis vulgaris ${ }^{1} ; \mathrm{KH}=$ Krankenhaus

${ }^{1}$ Mittelwerte der Patienten, die mindestens ein Mal beim Arzt gewesen waren.

${ }^{*} p<0,05$ vs. Befragte ohne Pso; ${ }^{\dagger} p<0,05$ vs. Patienten mit leichter Pso.

$¥$ Verlässliche statistische Schlussfolgerungen konnten aufgrund der geringen Patientenzahl $(<30)$ nicht gezogen werden. 
Tab. 3 Jährliche patientenseitige finanzielle Belastungen („Out-of-pocket-payments“) (in €).

\begin{tabular}{|c|c|c|c|c|c|}
\hline & Keine Pso & Pso gesamt & Leichte Pso & $\begin{array}{l}\text { Mittelschwere/ } \\
\text { Schwere Pso }\end{array}$ & Schwere Pso \\
\hline Alle Befragten, Mittelwert & 464 & 757 & 661 & $988^{*}$ & 2466 \\
\hline Nur Befragte mit Zuzahlungen, Mittelwert & 735 & 994 & 889 & $1249^{*}$ & 2785 \\
\hline
\end{tabular}

Befragte aus der Gruppe der mittelschwer/schwer Betroffenen gingen fast doppelt so häufig zum Arzt wie Personen ohne Pso (13 vs. 7 Arztbesuche; $p<0,05$ ).

Zwischen den Angaben der leicht und mittelschwer/schwer Betroffenen zeigten sich statistisch signifikante Unterschiede sowohl bzgl. des Anteils der Patienten, die einen Dermatologen konsultiert hatten, als auch bzgl. der Häufigkeit dieser Besuche innerhalb der letzten sechs Monate vor der Befragung (24\% mit 2 Besuchen vs. 38\% mit 5 Besuchen; p <0,05). Ähnliche Unterschiede finden sich für Besuche beim Rheumatologen ( $5 \%$ mit 3 Besuchen vs. $13 \%$ mit 4 Besuchen; $\mathrm{p}<0,05$ ).

In Bezug auf den Anteil der Befragten mit Krankenhausaufenthalten lassen sich insgesamt keine signifikanten Unterschiede zwischen den Gruppen aufzeigen. Im Fall einer Einweisung ist die durchschnittliche Verweildauer in der Gruppe der mittelschwer/ schwer Betroffenen allerdings höher (10 vs. 7,6 Tage bei leichter Pso).

\section{Patientenseitige Kosten \\ $\nabla$}

Unter „Out-of-pocket-payments“ versteht man direkte patientenseitige Kosten wie z.B. Zuzahlungen zu ärztlichen Leistungen und Medikamenten sowie Aufwendungen für rezeptfreie Medikamente und Hilfsmittel. Erfragt wurden nicht nur die Pso-spezifischen, sondern alle den Befragten entstehenden patientenseitigen Krankheitskosten. So gaben Befragte mit mittelschwerer/ schwerer Pso signifikant höhere Ausgaben im Vergleich zu den Nicht-Betroffenen an (jährlich durchschnittlich $988 €$ vs. $464 €$; p $<0,05-$ Tab. 3).

Betrachtet man in der Gruppe der Befragten mit mittelschwerer/ schwerer Pso nur diejenigen, bei denen überhaupt solche Ausgaben anfielen, so beliefen sich die jährlichen Kosten auf durchschnittlich $1249 €$ im Vergleich zu $735 €$ bei den Nicht-Betroffenen $(p<0,05)$. Bei den schwer Betroffenen lagen diese Kosten sogar bei $2785 €$.

\section{Produktivität \\ $\nabla$}

Grundsätzlich war bei allen Befragten mit Pso das Arbeitsleben in Form von Arbeitsausfall und Produktivitätsverlust beeinträchtigt. Bereits die Studienteilnehmer mit leichter Pso wiesen im Fragebogen WPAI signifikant erhöhte Einschränkungen im Arbeitsleben sowie in ihren alltäglichen Aktivitäten auf ( $\bullet$ Tab.4).

Diese Einbußen nahmen mit steigendem Schweregrad der Pso zu. Besonders stark schlug sich eine Beeinträchtigung in verringerter Produktivität am Arbeitsplatz nieder, die bei den Befragten mit mittelschwerer/schwerer und leichter Pso signifikant höher ausfiel als in der Gruppe der Nicht-Betroffenen (23,4\% und 20,6\% vs. $15,1 \%$; $<$ 0,05). Bei den Befragten mit schwerer Pso fiel die
Beeinträchtigung sogar doppelt so hoch aus wie in der Gruppe der Nicht-Betroffenen (31,9\% vs. $15,1 \%$ ).

\section{Lebensqualität \\ $\nabla$}

Die körperbezogene LQ war bei den Pso-Patienten im Vergleich zu den Nicht-Betroffenen signifikant vermindert (PCS von 42,2 vs. 47,6 Punkte; $\mathrm{p}<0,05$ ) ( Tab. 5).

Bei den Patienten mit mittelschwerer/schwerer Pso war auch die psychische Belastung signifikant erhöht (MCS von 43 für mittelschwere/schwere Pso vs. 49 in der Gruppe der Befragten ohne Pso und bei den leicht Betroffenen; $\mathrm{p}<0,05)$. In der Gruppe der schwer Betroffenen war die LQ in beiden Dimensionen am stärksten reduziert (MCS von 38 vs. 49 bzw. PCS von 42 vs. 48 bei den schwer bzw. den Nicht-Betroffenen).

\section{Diskussion}

Ziel der vorliegenden Studie war es, auf der Grundlage der internetbasierten NHWS Patienten mit Psoriasis in einer populationsbezogenen Stichprobe bezüglich medizinischer Ressourcennutzung, Arbeitsproduktivität und gesundheitsbezogener LQ zu charakterisieren und unterschiedliche Schweregrade der Pso in Hinblick auf diese Dimensionen miteinander und mit Nicht-Betroffenen zu vergleichen. Aufgrund des bevölkerungsbezogenen Charakters der NHWS umfasst die Auswertung einen näherungsweise repräsentativen Querschnitt der Bevölkerung. Damit wird eine methodische Lücke geschlossen, da der Einschluss der Studienteilnehmer nicht wie sonst üblich in den entsprechenden dermatologischen Zentren bzw. Hautarztpraxen erfolgte.

Für die Validität der vorliegenden Daten aus der NHWS spricht die fast exakte Übereinstimmung der Pso-Prävalenz von knapp $2,5 \%$ mit den für Deutschland publizierten Daten. Diese Angabe findet sich auch in den populationsbezogenen GKV-Daten von Augustin et al [3]. Sie korrespondieren auch mit der von Schäfer et al. publizierten Punktprävalenz der Pso von 2,1\% [28].

Auf Basis der NHWS Daten leiden 28\% der Pso-Betroffenen an einer mittelschweren oder schweren Pso. Diese Größenordnung entspricht anderen aktuellen Forschungsergebnissen [20,32].

Bezüglich der mit Pso assoziierten Begleiterkrankungen ergaben die NHWS-Daten wie auch die bisher verfügbaren epidemiologischen Komorbiditätsdaten [3] einen signifikant höheren Anteil an Komorbidität bei den Pso-Patienten. Hier ist insbesondere die PsA zu erwähnen, deren Bedeutung mit einer Prävalenz von knapp $20 \%$ bei den Pso-Betroffenen auch von anderen Studien hervorgehoben wird [21,24].

Neben einer erhöhten Inanspruchnahme des Gesundheitssystems (z.B. Arztbesuche) fielen bei den NHWS-Daten auch beträchtliche Kosten für die Patienten selbst auf. Darüber hinaus 
Tab. 4 Beeinträchtigung der Arbeitsaktivität (WPAl); höhere Werte weisen auf verminderte Produktivität/Aktivität hin.

\begin{tabular}{|c|c|c|c|c|c|}
\hline WPAI Komponenten (in \%) & Keine Pso & Pso gesamt & Leichte Pso & $\begin{array}{l}\text { Mittelschwere/ } \\
\text { Schwere Pso }\end{array}$ & Schwere Pso $\ddagger$ \\
\hline Absenteeism, Mittelwert & 4,9 & 4,9 & 5,2 & 4,1 & 5,5 \\
\hline Presenteeism, Mittelwert & 15,1 & $21,4^{*}$ & $20,6^{*}$ & $23,4^{*}$ & 31,9 \\
\hline TWPI, Mittelwert & 18,1 & $24,1^{*}$ & $23,6^{*}$ & $25,1^{*}$ & 33,3 \\
\hline TAI, Mittelwert & 24,4 & $33,1^{*}$ & $32,4^{*}$ & $35,0^{*}$ & 35,0 \\
\hline \multicolumn{6}{|c|}{$\begin{array}{l}\text { WPAI (Work Productivity and Activity Impairment) = Fragebogen zu Arbeitsausfall und Produktivitätsverlust; Pso = Psoriasis vulgaris; Absenteeism = krankheitsbe- } \\
\text { dingte Fehlstunden geteilt durch übliche Arbeitsstunden, bezogen auf die letzten } 7 \text { Tage; Presenteeism = krankheitsbedingter Produktivitätsverlust während der } \\
\text { Arbeitszeit, bezogen auf die letzten } 7 \text { Tage; TWPI (Total Work Productivity Index) = kombinierte Effekte von Absenteeism und Presenteeism, bezogen auf die üblichen } \\
\text { Arbeitsstunden der letzten } 7 \text { Tage; TAI (Total Activity Index) = Einschränkung der Fähigkeit, alltäglichen außerberuflichen Tätigkeiten nachzugehen, bezogen auf die } \\
\text { letzten } 7 \text { Tage. Alle Scores werden in Prozent dargestellt. In die Berechnung von Absenteeism, Presenteeism und TWPI werden nur berufstätige Patienten einge- } \\
\text { schlossen (zwischen } 56,5 \% \text { keine Pso bis } 45,1 \% \text { schwere Pso); in den TAI gehen die Daten aller Patienten ein. } \\
\text { p }<0,05 \text { vs. Befragte ohne Pso; } ¥ \text { Verlässliche statistische Schlussfolgerungen konnten aufgrund der geringen Patientenzahl (<30) nicht gezogen werden. }\end{array}$} \\
\hline
\end{tabular}

Tab. 5 Lebensqualität, gemessen mit dem SF-12: Körperliche (PCS) und psychische (MCS) Summenskalen.

\begin{tabular}{llllll} 
& Keine Pso & Pso gesamt & Leichte Pso & $\begin{array}{l}\text { Mittelschwere/ } \\
\text { Schwere Pso }\end{array}$ & \multicolumn{2}{c}{ Schwere Pso ${ }^{\ddagger}$} \\
\hline MCS, Mittelwert & 48,67 & 47,30 & 48,75 & $43,49^{*} \dagger$ & 38,28 \\
\hline PCS, Mittelwert & 47,63 & $42,21^{*}$ & $42,95^{*}$ & $42,90^{*}$ & 41,88
\end{tabular}

SF-12 (Short Form 12) = Fragebogen zur gesundheitsbezogenen Lebensqualität; PCS (Physical Component Score) = körperliche Dimension der gesundheitsbezogenen Lebensqualität; MCS (Mental Component Score) = psychische Dimension der gesundheitsbezogenen Lebensqualität; Pso = Psoriasis vulgaris

${ }^{*} \mathrm{p}<0,05$ vs. Befragte ohne Pso; ${ }^{\dagger} \mathrm{p}<0,05$ vs. Patienten mit leichter Pso.

$¥$ Verlässliche statistische Schlussfolgerungen konnten aufgrund der geringen Patientenzahl $(<30)$ nicht gezogen werden.

wurden eine eingeschränkte gesundheitsbezogene LQ und eine beeinträchtigte Arbeitsproduktivität angegeben.

Bezüglich der Inanspruchnahme von medizinischen Ressourcen wurde ermittelt, dass mittelschwer/schwer Betroffene fast doppelt so häufig einen Arzt aufsuchten wie Befragte ohne Pso. Berger et al. ermittelten in ihrer Gesundheitskostenstudie durchschnittlich 7,2 Besuche beim Dermatologen pro Jahr und Patient [5], während die NHWS für einen Zeitraum von sechs Monaten fünf Arztbesuche pro Patient konstatiert. Zu diesen Belastungen für das Gesundheitswesen müssen noch die Ausgaben für die Patienten selbst hinzugerechnet werden. Diese beliefen sich in der Gruppe der mittelschwer/schwer Betroffenen auf durchschnittlich $988 €$ im Vergleich zu $464 €$ bei Personen ohne Pso. Berger et al. ermittelten durchschnittliche „Out-of-pocket-payments“ von $562 €$ pro Jahr und Patient [5], eine weitere Gesundheitskostenstudie bezifferte diese Ausgaben mit $794 €$ [30].

Bei den Patienten mit schwerer Pso gibt es zwar keinen Unterschied in der Häufigkeit der Krankenhausaufenthalte im Vergleich zu den anderen Gruppen; im Falle einer Einweisung wurde aber ein längerer Aufenthalt ermittelt. Diesen Zahlen liegt möglicherweise ein Bias zugrunde, da in Deutschland viele Patienten mit schwerer Pso nicht nur ins Krankenhaus eingewiesen werden, sondern sich auch häufig in Rehabilitationsmaßnahmen befinden. Diese betreffen dann auch die vom Krankenkassensystem unabhängige Rentenversicherung. Die Dimension dieser Kosten wird nicht widergespiegelt, da im Rahmen der NHWS keine Daten zu Reha-Maßnahmen abgefragt wurden. Gemäß publizierter Daten beträgt die durchschnittliche Verweildauer für Pso-Patienten in Reha-Einrichtungen in Deutschland ca. 25 Tage [19], an denen zusätzlich Kosten anfallen und in denen berufstätige Patienten arbeitsunfähig sind.

Arbeitsausfall und krankheitsbedingt verminderte Leistungsfähigkeit, die in der NHWS deutlich zu Tage traten, verursachen Produktivitätsverluste und führen daher ebenfalls zu einer beträchtlichen volkswirtschaftlichen Belastung. Bei Sohn et al. bzw. Schöffski et al. beliefen sich diese indirekten Kosten auf $20 \%$ der durch Pso entstandenen Gesamtlast [30,32], während dieser Anteil an den Gesamtkosten bei Berger et al. sogar etwas mehr als die Hälfte aller entstandenen Kosten darstellte [5]. In den letzten Jahren sind neben den direkt quantifizierbaren Kosten auch die intangiblen Kosten in den Vordergrund gerückt, die durch die Beeinträchtigung der gesundheitsbezogenen LQ entstehen. In der vorliegenden Analyse mit einem generischen LQ-Fragebogen war in der Gesamtgruppe der Pso-Patienten die körperliche Dimension der LQ signifikant vermindert, bei den Befragten mit mittelschwerer/schwerer Pso auch die psychische. Der Befund einer verminderten LQ bei Psoriasis bestätigt ausnahmslos die bislang publizierten nationalen und internationalen Vorarbeiten $[2,4,7,12,13,22,29,30,36]$.

In der vorliegenden Arbeit wurde ebenfalls bestätigt, dass die LQ auch bei geringer Flächenausdehnung bereits stark vermindert sein kann. In der Literatur wurde dies beispielsweise bei Befall intimer oder exponierter Körperstellen postuliert [30,27]. Bei Zachariae et al. war eine Beeinträchtigung der gesundheitsbezogenen LQ der stärkste Prädiktor für psychische Erkrankungen wie Depression, Angststörung und Suizidalität [36]. Die durch Pso hervorgerufene Krankheitslast liegt dabei in einer Größenordnung wie die Belastungen durch chronische internistische Krankheiten und Tumorleiden [23]. Daran lässt sich erkennen, dass die rein morphologische Beurteilung der Pso die tatsächliche Krankheitslast nicht adäquat widerspiegelt.

Es ist daher von großer Bedeutung, dass der behandelnde Arzt sich dieser Belastungsdimensionen bewusst ist und sie bei der Beurteilung des Schweregrades der Krankheit und der Therapieentscheidung berücksichtigt. Daraus leitet sich das Therapieziel ab, nicht nur eine Verbesserung der kutanen Läsionen zu erreichen, sondern auch die Krankheitslast zu reduzieren sowie eine Verbesserung der LQ zu erzielen [17]. Die konsequente Verfolgung eines Behandlungsalgorithmus ist entscheidend, wie er mit der S3-Leitlinie für Deutschland entwickelt wurde [17]. Der darin bei mittelschwerer bis schwere Psoriasis vorgesehene Ein- 
satz von Systemtherapeutika und Biologika trägt zur Verbesserung der Lebensqualität entscheidend bei [16,20,26,31]. Eine Bilanzierung von Therapiekosten mit dem zu erzielenden Nutzen fällt deshalb nicht zuletzt auch unter dem Gesichtspunkt des Zugewinns an LQ und Produktivität positiv aus. Der Einsatz von innovativen Therapien wie Biologika ist somit nicht nur aus medizinischer und ethischer Sicht, sondern auch aus wirtschaftlicher Perspektive geboten [18,20,31].

Eine Stärke der vorliegenden Studie ist die bevölkerungsbezogene Erhebung unter Umgehung von verzerrenden Versorgungsstrukturen wie Praxen, Kliniken oder Apotheken. Dennoch können auch bei der vorliegenden webbasierten Erhebung potenzielle Effektmodifikatoren nicht ausgeschlossen werden, z. B. ein mit der Methode der Selbstauskunft einhergehenden „recall bias“ sowie ein Selektionsbias aufgrund der „Selbstauswahl“ der Teilnehmer. Eine weitere potenzielle methodische Fehlerquelle liegt in der Bestimmung des Schweregrades durch Selbsteinschätzung der Befragten mittels BSA [14], die allerdings auch in anderen Studien als gängige Methode verwendet wurde [15,33]. Aufgrund der direkten Befragung von Patienten im Rahmen des Surveys NHWS wurden nicht die gesamten direkten Ressourcenverbrauche ermittelt, sodass nicht die Gesamtkosten zu Lasten der GKV darstellbar sind. Dennoch haben sich die Daten - soweit mit Vorstudien vergleichbar - als inhaltlich valide und hinsichtlich der Schweregrad-Klassifikation als plausibel erwiesen.

\section{Fazit für die Praxis}

Als systemische und chronische Entzündungserkrankung führt Pso zu einer deutlichen Einschränkung der Lebensqualität und stellt aufgrund ihrer Häufigkeit und der Auswirkungen auf Produktivität und berufliche Aktivität eine hohe volkswirtschaftliche Belastung dar. Die medizinischen und ökonomischen Konsequenzen der Erkrankung erfordern eine frühe, qualifizierte Versorgung nach den Standards der S3-Leitlinie. Sie rechtfertigen den Verbrauch von Ressourcen für eine adäquate Therapie.

Zur Evaluierung des Schweregrades und zur Beurteilung des Therapieverlaufes sollten dabei nicht nur das Ausmaß des Hautbefalls berücksichtigt, sondern auch psychische, psychosoziale und ökonomische Parameter herangezogen werden. Die Behandlungsstrategien zielen über die Linderung der körperlichen Beschwerden hinaus auf die Verbesserung der gesamten Lebensqualität ab.

\section{Abstract}

\section{Psoriasis Causes High Costs, Reduces Productivity at Work and Quality of Life $\nabla$}

Data of the 2007 web-based NHWS (National Health and Wellness Survey) have been evaluated regarding the prevalence, utilisation of health care resources, work productivity and burden of disease in psoriasis (pso) patients compared to non-affected responders. Based on the NHWS, 382 out of 15000 respondents reported being diagnosed with pso by a physician. This reflects a prevalence of $2.5 \%$ and project to approximately 1.9 million adults affected by pso in Germany. Particularly patients with moderate/severe pso were found to take more advantage of the health care system, to have higher out-of-pocket-expenses and reduced work productivity. Health-related quality of life was substantially reduced. These aspects are important when deciding on the adequate therapy.

\section{Literatur}

1 Augustin M, Glaeske G, Radtke MA et al. Epidemiology and comorbidity of psoriasis in children. Br J Dermatol 2010; 162 (3): 633-636

2 Augustin M, Krüger K, Radtke MA, Schwippl I, Reich K. Disease Severity, Quality of Life and Health Care in Plaque-Type Psoriasis: A Multicenter Prospective Cross-Sectional Study in Germany. Dermatology 2008; 216: $366-372$

3 Augustin M, Reich K, Glaeske G, Schaefer I, Radtke M. Comorbidity and age-related prevalence of psoriasis - analysis of health insurance data in Germany. Acta Derm Venereol 2010; 90: 147-151

4 Augustin M, Reich K, Reusch M et al. Health services research in psoriasis - the German approach. Dermatology 2009; 218 (4): 293 - 301

5 Berger K, Ehlken B, Kugland B, Augustin M. Cost-of-illness in patients with moderate and severe chronic psoriasis vulgaris in Germany. J Dtsch Dermatol Ges 2005; 3 (7): 511 - 518

6 Bullinger M, Kirchberger I. SF-36. Fragebogen zum Gesundheitszustand. Göttingen: Hogrefe; 1998

7 Consumer Health Sciences (CHS). Im Internet:www.chsinternational. com Stand: 15.09.2009

8 Delfino MJr, Holt EW, Taylor CR, Wittenberg E, Qureshi AA. Willingnessto-pay stated preferences for 8 health-related quality-of-life domains in psoriasis: a pilot study. J Am Acad Dermatol 2008; 59 (3): 439-447

9 Jiménez-Puya R, Gómez-García F, Amorrich-Campos V, Moreno-Giménez JC. Etanercept: Efficacy and safety. J Eur Acad Dermatol Venereol 2009; 23 (4): $402-405$

10 Gelfand JM, Troxel AB, Lewis JD et al. The risk of mortality in patients with psoriasis: results from a population-based study. Arch Dermatol 2007; 143 (12): $1493-1499$

11 Griffiths CEM, Camp RDR, Barker JNWN. Rook's textbook of dermatology. 7th ed. Oxford: Blackwell; 2005

12 Kimball $A B$, Jacobson $C$, Weiss $S$, Vreeland MG, $W u$ Y. The psychosocial burden of psoriasis. Am J Clin Dermatol 2005; 6 (6): 383-92

13 Krüger G, Koo J, Lebwohl $M$ et al. The impact of psoriasis on quality of life: results of a 1998 National Psoriasis Foundation patient-membership survey. Arch Dermatol 2001; 137 (3): 280-284

14 Krüger GG, Feldman SR, Camisa C et al. Two considerations for patients with psoriasis and their clinicians: what defines mild, moderate, and severe psoriasis? What constitutes a clinically significant improvement when treating psoriasis? J Am Acad Dermatol 2000; 43 (2 Pt 1): $281-5$

15 Kurd SK, Gelfand JM. The prevalence of previously diagnosed and undiagnosed psoriasis in US adults: results from NHANES 2003-2004. 2009; 60 (2): 218-24

16 Mrowietz U, Elder JT, Barker J. The importance of disease associations and concomitant therapy for the long-term management of psoriasis patients. Arch Dermatol Res 2006; 298 (7): 309-319

17 Nast A et al. S3-Leitlinie zur Therapie der Psoriasis vulgaris. J Dtsch Dermatol Ges 2011

18 Nelson AA, Pearce DJ, Fleischer ABJr, Balkrishnan R, Feldman SR. Cost-effectiveness of biologic treatments for psoriasis based on subjective and objective efficacy measures assessed over a 12-week treatment period. J Am Acad Dermatol 2008; 58 (1): 125- 35

19 Official figures by the Federal Statistical Bureau on average stays in rehabilitation care in the ICD 10 code L40. Im Internet: http://www. gbe-bund.de/oowa921-install/servlet/oowa/aw92/dboowasys921. xwdevkit/xwd_init?gbe.isgbetol/xs_start_neu/i95479111/47809735 Stand: 15.09 .2009

20 Radtke MA, Augustin M. Effiziente Kontrolle erforderlich. Kosten und Wirtschaftlichkeit der Psoriasis-Therapie. Gesellschaftspolitische Kommentare 2008; 1: 26-29

21 Radtke MA, Reich K, Blome C, Rustenbach S, Augustin M. Prevalence and clinical features of psoriatic arthritis and joint complaints in 2009 patients with psoriasis: results of a German national survey. J Eur Acad Dermatol Venereol 2009; 23 (6): 683-91

22 Radtke M, Reich K, Reich C et al. Evaluation of quality of care and guideline-compliant treatment in psoriasis: development of a new system of quality indicators. Dermatology 2009; 219 (1): 54-8

23 Rapp SR, Feldman SR, Exum ML, Fleischer AB Jr, Reboussin DM. Psoriasis causes as much disability as other major medical diseases. J Am Acad Dermatol 1999; 41 (3 Pt 1): $401-7$ 
24 Reich K, Krüger K, Mössner R, Augustin M. Epidemiology and clinical pattern of psoriatic arthritis in Germany: a prospective interdisciplinary epidemiological study of 1511 patients with plaque-type psoriasis. Br J Dermatol 2009; 160 (5): 1040-7

25 Reilly MC, Zbrozek AS, Dukes EM. The validity and reproducibility of a work productivity and activity impairment instrument. Pharmacoeconomics 1993; 4 (5): $353-365$

26 Revicki DA, Menter A, Feldman S et al. Adalimumab improves healthrelated quality of life in patients with moderate to severe plaque psoriasis compared with the United States general population norms: results from a randomized, controlled Phase III study. Health Qual Life Outcomes 2008; 6: 75

27 Schäfer I, Hacker J, Rustenbach SJ et al. Concordance of the Psoriasis Area and Severity Index (PASI) and patient-reported outcomes in psoriasis treatment. Eur J Dermatol 2010; 20 (1): 62 - 67

28 Schäfer I, Rustenbach SJ, Zimmer L, Augustin M. Prevalence of skin diseases in a cohort of 48,665 employees in Germany. Dermatology 2008; 217 (2): $169-172$

29 Schmid-Ott G, Künsebeck HW, Jäger B et al. Significance of the stigmatization experience of psoriasis patients: a 1-year follow-up of the illness and its psychosocial consequences in men and women. Acta Derm Venereol 2005; 85 (1): 27-32
30 Schöffski O, Augustin M, Prinz J et al. Costs and quality of life in patients with moderate to severe plaque-type psoriasis in Germany: a multicenter study. J Dtsch Dermatol Ges 2007; 5 (3): 209-218

31 Sizto S, Bansback N, Feldman SR, Willian MK, Anis AH. Economic evaluation of systemic therapies for moderate to severe psoriasis. Br J Dermatol 2009; 160 (6): $1264-72$

32 Sohn S, Schöffski O, Prinz J et al. Cost of moderate to severe plaque psoriasis in Germany: a multicenter cost-of-illness study. Dermatology 2006; 212 (2): 137 - 144

33 Stern RS, Nijsten T, Feldman SR et al. Psoriasis is common, carries a substantial burden even when not extensive, and is associated with widespread treatment dissatisfaction. J Investig Dermatol Symp Proc 2004; 9: $136-139$

34 United States Census Bureau and Organization for Economic Cooperation and Development, International Database (2007). Im Internet: http://www.census.gov Stand: 20.08.2009

35 Ware JE, Kosinski M, Turner-Bowker DM, Gandek B. How to score Version 2 of the SF- $12^{\circledR}$ Health Survey (with a supplement documenting version 1). Health Assessment Lab 2002

36 Zachariae R, Zachariae C, Ibsen HH, Mortensen JT, Wulf HC. Psychological symptoms and quality of life of dermatology outpatients and hospitalized dermatology patients. 2002; 84 (3): 205-212 